\title{
Gollop-Wolfgang complex
}

INSERM

\section{Source}

INSERM. (1999). Orphanet: an online rare disease and orphan drug data base. GollopWolfgang complex. ORPHA:1986

Gollop-Wolfgang complex is a very rare malformation characterized by ectrodactyly of the hand and ipsilateral bifurcation of the femur. 